\title{
The carpometacarpal joint of the thumb: MR appearance in asymptomatic volunteers
}

\author{
Hirschmann, Anna ; Sutter, Reto ; Schweizer, Andreas ; Pfirrmann, Christian W A
}

\begin{abstract}
PURPOSE: To prospectively characterize the MR appearance of the carpometacarpal (CMC) joint of the thumb in asymptomatic volunteers. MATERIALS AND METHODS: Thirty-four asymptomatic volunteers (17 women, 17 men, mean age, $33.9 \pm 9.2$ years) underwent MR imaging of the thumb after approval by the local ethical committee. Two musculoskeletal radiologists independently classified visibility and signal intensity (SI) characteristics of the anterior oblique (AOL/beak ligament), the posterior oblique (POL), the intermetacarpal (IML), and the dorsoradial ligaments (DRL) on a threepoint Likert scale. The thickness of all ligaments, cartilage integrity, and presence of joint fluid were assessed. The alignment of the first metacarpal base with the trapezium was quantified on sagittal and coronal planes. RESULTS: The ligaments of the CMC joint were constantly visible in all volunteers for the POL and IML, and in all but one for the AOL and DRL. On intermediate-weighted fat-saturated images the POL $(65 \% / 74 \%$ reader $1 /$ reader 2$)$ and DRL $(58 \% / 64 \%)$ were commonly of increased SI, while the IML had a striated appearance in $91 \% / 76 \%$ of subjects. The AOL showed a variable SI (36 $\% / 42 \%$ low, $27 \% / 27 \%$ increased, $36 \% / 30 \%$ striated). The IML was the thickest ligament with a mean of $2.9 \mathrm{~mm} / 3.1 \mathrm{~mm}$ and the DRL the thinnest $(1.2 \mathrm{~mm} / 1.4 \mathrm{~mm})$. There was a mean dorsal subluxation of $1.8 \mathrm{~mm} / 2.0 \mathrm{~mm}$ and radial subluxation of $2.8 \mathrm{~mm} / 3.4 \mathrm{~mm}$ of the metacarpal base. The AOL was significantly thicker in men $(1.7 \mathrm{~mm})$ than in women $(1.2 \mathrm{~mm} ; \mathrm{p}=0.02)$. Radial subluxation was significantly larger in men $(3.4 \mathrm{~mm})$ than in women $(2.2 \mathrm{~mm} ; \mathrm{p}=0.02)$. No subluxation in palmar or ulnar direction was seen. CONCLUSIONS: Radial and dorsal subluxation of the CMC joint can be a normal finding in a resting position at MR imaging. The CMC ligaments showed a considerable variability of signal intensity with a typically striated IML; thickness of the AOL is typically less than $2.2 \mathrm{~mm}$, of the POL typically less than $2.9 \mathrm{~mm}$.
\end{abstract}

DOI: https://doi.org/10.1007/s00256-013-1633-4

Posted at the Zurich Open Repository and Archive, University of Zurich

ZORA URL: https://doi.org/10.5167/uzh-86645

Journal Article

Published Version

Originally published at:

Hirschmann, Anna; Sutter, Reto; Schweizer, Andreas; Pfirrmann, Christian W A (2013). The carpometacarpal joint of the thumb: MR appearance in asymptomatic volunteers. Skeletal Radiology, 42(8):1105-1112.

DOI: https://doi.org/10.1007/s00256-013-1633-4 


\title{
The carpometacarpal joint of the thumb: MR appearance in asymptomatic volunteers
}

\author{
Anna Hirschmann • Reto Sutter • Andreas Schweizer • \\ Christian W. A. Pfirrmann
}

Received: 23 January 2013 /Revised: 1 April 2013 /Accepted: 21 April 2013 /Published online: 15 May 2013

(C) ISS 2013

\begin{abstract}
Purpose To prospectively characterize the MR appearance of the carpometacarpal (CMC) joint of the thumb in asymptomatic volunteers.

Materials and methods Thirty-four asymptomatic volunteers (17 women, 17 men, mean age, $33.9 \pm 9.2$ years) underwent MR imaging of the thumb after approval by the local ethical committee. Two musculoskeletal radiologists independently classified visibility and signal intensity (SI) characteristics of the anterior oblique (AOL/beak ligament), the posterior oblique (POL), the intermetacarpal (IML), and the dorsoradial ligaments (DRL) on a three-point Likert scale. The thickness of all ligaments, cartilage integrity, and presence of joint fluid were assessed. The alignment of the first metacarpal base with the trapezium was quantified on sagittal and coronal planes.

Results The ligaments of the CMC joint were constantly visible in all volunteers for the POL and IML, and in all but one for the AOL and DRL. On intermediate-weighted fat-saturated images the POL $(65 \% / 74 \%$ reader $1 /$ reader 2$)$ and DRL (58\%/64 \%) were commonly of increased SI, while the IML had a striated appearance in $91 \% / 76 \%$ of
\end{abstract}

\footnotetext{
A. Hirschmann $(\bowtie) \cdot$ R. Sutter $\cdot$ C. W. A. Pfirmann Department of Radiology, Orthopedic University Hospital Balgrist, University of Zurich, Forchstrasse 340, 8008 Zurich, Switzerland e-mail: anna.hirschmann@balgrist.ch

R. Sutter

e-mail: reto.sutter@balgrist.ch

C. W. A. Pfirmann

e-mail: christian.pfirrmann@balgrist.ch

\section{A. Schweizer}

Department of Orthopedic Surgery, Orthopedic University Hospital Balgrist, University of Zurich, Forchstrasse 340, 8008 Zurich, Switzerland

e-mail: andreas.schweizer@balgrist.ch
}

subjects. The AOL showed a variable SI (36\%/42 \% low, $27 \% / 27 \%$ increased, $36 \% / 30 \%$ striated). The IML was the thickest ligament with a mean of $2.9 \mathrm{~mm} / 3.1 \mathrm{~mm}$ and the DRL the thinnest $(1.2 \mathrm{~mm} / 1.4 \mathrm{~mm})$. There was a mean dorsal subluxation of $1.8 \mathrm{~mm} / 2.0 \mathrm{~mm}$ and radial subluxation of $2.8 \mathrm{~mm} / 3.4 \mathrm{~mm}$ of the metacarpal base. The AOL was significantly thicker in men $(1.7 \mathrm{~mm})$ than in women $(1.2 \mathrm{~mm} ; p=0.02)$. Radial subluxation was significantly larger in men $(3.4 \mathrm{~mm})$ than in women $(2.2 \mathrm{~mm} ; p=0.02)$. No subluxation in palmar or ulnar direction was seen.

Conclusions Radial and dorsal subluxation of the CMC joint can be a normal finding in a resting position at MR imaging. The CMC ligaments showed a considerable variability of signal intensity with a typically striated IML; thickness of the AOL is typically less than $2.2 \mathrm{~mm}$, of the POL typically less than $2.9 \mathrm{~mm}$.

Keywords Carpometacarpal joint · Thumb · Magnetic resonance imaging

\section{Introduction}

The carpometacarpal (CMC) joint of the thumb is a saddle joint with a biconcave-convex shape. The trapezium articulates with the first and second metacarpal base distally [1]. This unique morphology leads to a wide range of motion [2-4]. Stability is given by a ligament complex, which consists of five main components (Fig. 1): the anterior oblique ligament (AOL/beak ligament), the posterior oblique ligament (POL), the dorsoradial ligament (DRL), the intermetacarpal ligament (IML) and the ulnar collateral ligament (UCL) [2, 3, $5,6]$. Due to the exceptional anatomy of the joint, it is lax and incongruent in the resting thumb position while it is stable and tightly congruent in the final phase of thumb opposition $[4,6,7]$. 


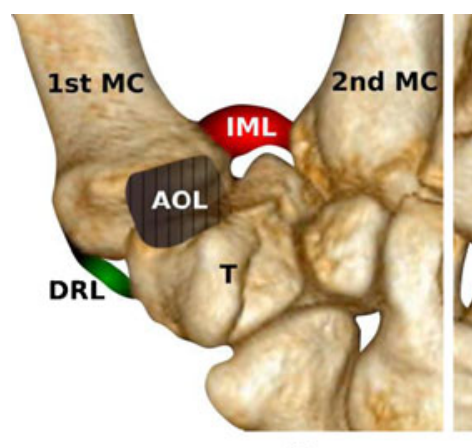

a

Fig. 1 3D reconstructions show the volar view (a), the dorsal view (b), and the radial view (c) of the carpometacarpal (CMC) ligament complex of the thumb. The dark (AOL) and white (POL) stripes demonstrate the ligament orientation. $\mathrm{AOL}=$ anterior oblique ligament/beak,

Isolated dislocation of the CMC joint is uncommon and occurs in less than $1 \%$ of all hand injuries $[6,8]$. It is more often combined with a fracture of the first metacarpal base $[7,9]$. A pure dislocation of the CMC joint typically leads to a rupture of the DRL and POL [6-8]. Magnetic resonance (MR) imaging can be used to accurately evaluate these ligament injuries [5].

The ligamentous anatomy of the $\mathrm{CMC}$ joint has been extensively studied in cadaveric dissections [1, 10-18]. Normal CMC ligament appearance on MR images is often described as low signal intensity (SI) with a thin linear shape in all sequences [3,5], although there are reports of variable SI in asymptomatic healthy volunteers in elbow and ankle ligaments $[19,20]$. Only a few studies have addressed the complex MR anatomy of the CMC ligaments in detail $[3,5]$. Also, the spectrum of normal MR morphology of the CMC joint structures in healthy volunteers is not known. The purpose of this study was to prospectively characterize the $\mathrm{MR}$ anatomy and variants of the $\mathrm{CMC}$ joint in asymptomatic volunteers.

\section{Materials and methods}

Thirty-four asymptomatic healthy volunteers (17 women, mean age 33.5 \pm 9.8 years, range, 20-49 years; 17 men, mean age $34.4 \pm 8.8$ years, range, $23-49$ years) were prospectively included in this study. For each decade between 20 and 50 years, at least five women and five men were included. All volunteers completed a questionnaire to ensure that they had not undergone prior thumb and hand surgery, had no thumb and hand pain, had never seen a physician for thumb and hand complaints, had never injured the thumb and hand and did not suffer from a systemic inflammatory disease. Daily and labor activities of the thumb were evaluated as well as the handedness. This study was approved by the local ethics committee and informed consent was obtained from each volunteer.

\section{MR imaging protocol}

MR imaging was performed with a 1.5-T extremity scanner (Optima MR 430 s, GE Healthcare, Waukesha, WI, USA). All subjects were examined in the static resting position of the thumb in a dedicated $100-\mathrm{mm}$ coil. The patient was sitting next to the extremity scanner, the arm was placed in the neutral position, the thumb rested parallel to the second digit. Intermediateweighted fat-saturated (FS) images were acquired in the sagittal plane (repetition time ms/echo time ms, 2,000/24; section thickness, $2 \mathrm{~mm}$; field of view, $67 \mathrm{~mm}$; acquisition time, $5 \mathrm{~min} 24 \mathrm{~s})$, in the coronal plane $(2,039 / 25$; section thickness, $2 \mathrm{~mm}$; field of view, $135 \mathrm{~mm}$; acquisition time, $5 \mathrm{~min} 29 \mathrm{~s}$ ) and in the axial plane perpendicular to the first metacarpal bone $(2,079 / 22$; section thickness, $2.5 \mathrm{~mm}$; field of view, $60 \mathrm{~mm}$; acquisition time, $5 \mathrm{~min} 48 \mathrm{~s}$ ). T1-weighted turbo spin echo images were obtained in the sagittal plane (598/14; section thickness, $2 \mathrm{~mm}$; field of view, $67 \mathrm{~mm}$; acquisition time, $4 \mathrm{~min}$ $55 \mathrm{~s})$ and in the axial plane perpendicular to the first metacarpal bone (557/13; section thickness, $2.5 \mathrm{~mm}$; field of view, $60 \mathrm{~mm}$; acquisition time, $5 \mathrm{~min} 5 \mathrm{~s}$ ).

Definition of ligaments

\section{Anterior oblique/beak ligament (AOL)}

The AOL has its origin at the palmar tubercle of the trapezium and attaches at the volar beak of the first metacarpal base (Figs. 1 and 2). The AOL runs in a 
Fig. 2 a Sagittal intermediateweighted fat-saturated image $(2,000 / 24 \mathrm{~ms})$ shows the AOL (black arrows) and the POL (white arrows) of the right $\mathrm{CMC}$ joint of the thumb in a 48 -yearold healthy volunteer. $\mathbf{b}$

Schematic drawing of the AOL and POL at the same position as a. $\mathrm{AOL}=$ anterior oblique ligament/beak, $\mathrm{POL}=$ posterior oblique ligament, 1 st $\mathrm{MC}=$ first metacarpal bone
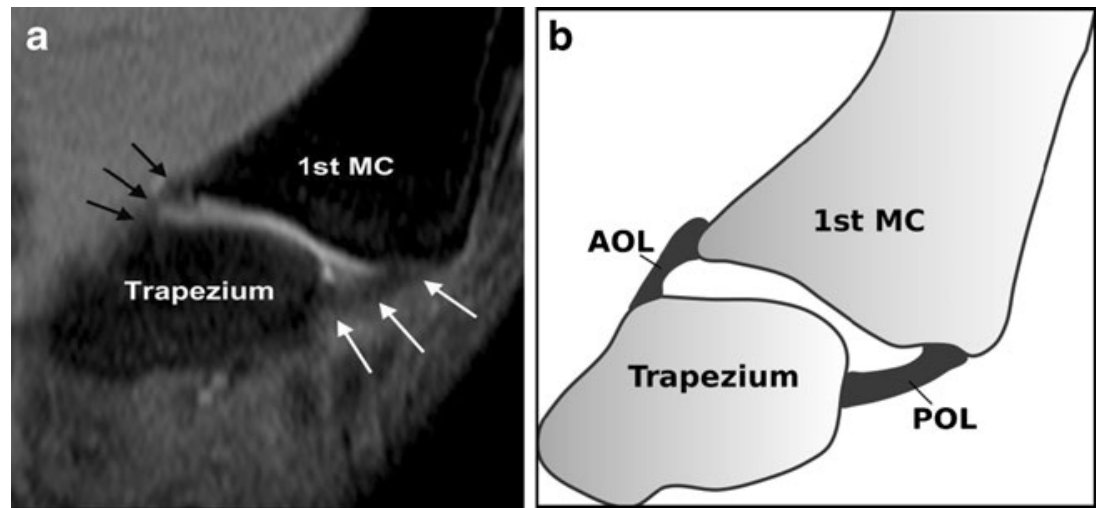

sagittal orientation. The ligament spans volarly around the $\mathrm{CMC}$ joint and runs in a diagonal fashion from proximal-radial to distal-ulnar.

The AOL is composed of two layers, a superficial and a deeper intraarticular one. Most authors do not differentiate between these two layers and term the AOL "beak ligament" $[4,7,10,17,21]$, while a few authors name the deep bundles "beak ligament" [3, 16$]$.

\section{Posterior oblique ligament (POL)}

The POL runs obliquely from the dorsal tubercle of the trapezium to the dorsoulnar tubercle of the metacarpal base (Figs. 1 and 2). Best visualization is in the sagittal imaging plane.

\section{Dorsoradial ligament (DRL)}

The DRL origins at the dorsoradial tubercle of the trapezium, attaches to the adjacent aspect of the metacarpal base and has a coronal orientation (Figs. 1 and 3).

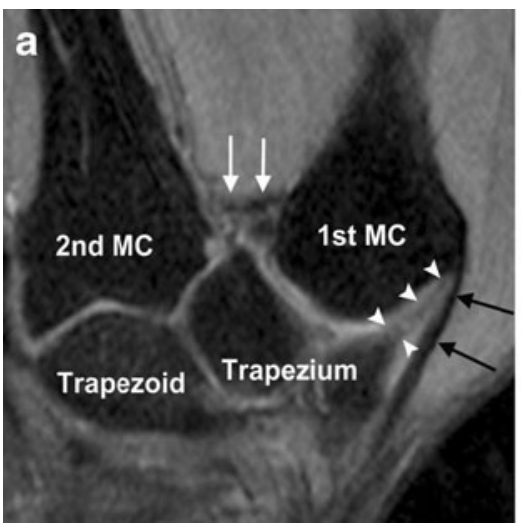

Fig. 3 a Coronal intermediate-weighted fat-saturated image (2,039/ $25 \mathrm{~ms}$ ) shows the DRL (arrowheads), IML (white arrows), and abductor pollicis longus tendon (black arrows) of the CMC joint of the thumb in a 33-year-old healthy volunteer. b Schematic drawing of
Intermetacarpal ligament (IML)

This extracapsular ligament arises from the radial base of the second metacarpal bone and runs transversally to the ulnar side of the first metacarpal base (Figs. 1 and 3). It is best visualized in coronal images.

\section{Qualitative analysis}

MR images were analyzed independently by two fellowshiptrained musculoskeletal radiologists. They were blinded to the results of each other's readings and to any information regarding the volunteers.

Visibility (no/yes) and SI (low as the normal low signal of ligaments and tendons/increased compared to the normal low signal of ligaments and tendons/striated) of the ligament complex (AOL, POL, DRL, IML) were evaluated on fluidsensitive intermediate-weighted FS images. The cartilage (intact/inhomogeneous SI/presence of chondral defects) as well as the presence of joint fluid (no/minor/joint distension) was assessed.

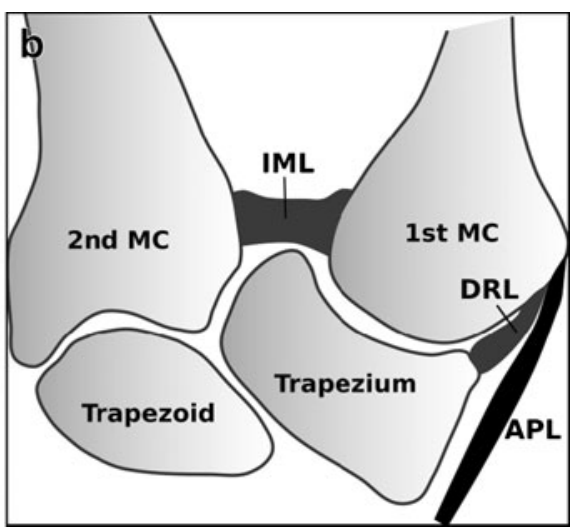

the DRL and IML at the same position as a. 1 st $\mathrm{MC}=$ first metacarpal bone, $2 \mathrm{nd} \mathrm{MC}=$ second metacarpal bone, $\mathrm{AOL}=$ anterior oblique ligament/beak, $\mathrm{POL}=$ posterior oblique ligament, $\mathrm{APL}=$ abductor pollicis longus tendon 

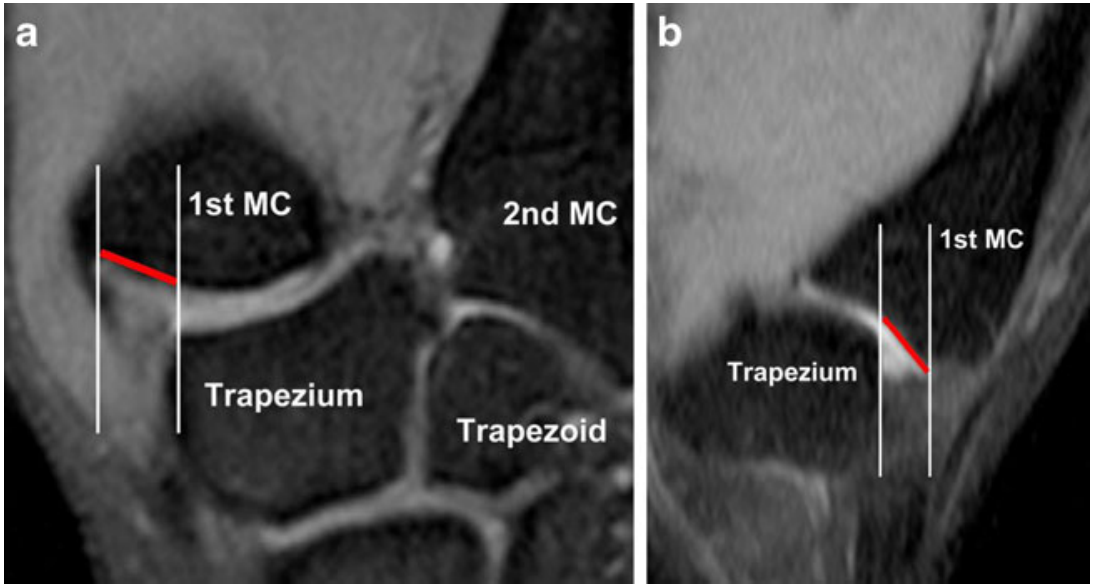

Fig. 4 a Coronal $(2,039 / 25 \mathrm{~ms})$ and b sagittal $(2,000 / 24 \mathrm{~ms})$ intermediate-weighted fat-saturated images show radial subluxation (a) and dorsal subluxation (b) of the metacarpal base of the right CMC joint of the thumb in a 33-year-old healthy volunteer. The white lines reference the

\section{Quantitative analysis}

Thickness of the ligaments was measured at the midportion of each ligament (AOL, POL, IML, and DRL). For alignment of the CMC joint, we measured the distance between the cartilage-covered portions parallel to the joint surface of the base of the first metacarpal bone and the trapezium (Fig. 4). This measurement was performed in the coronal and sagittal planes.

\section{Statistical analysis}

Descriptive statistics were used to report the quantitative and qualitative data of the CMC joint. Inter-rater agreement was assessed using kappa ( $K$ ) statistics for qualitative data and the intraclass correlation coefficient (ICC) for quantitative data. According to Landis and Koch [22], a $\mathrm{K}$ value of $0-0.2$ is indicative of slight agreement, $0.21-0.4$ fair cartilage-covered parts of the joint, the red lines show the measurement along the joint surfaces to assess subluxation distances. $1 \mathrm{st} \mathrm{MC}=$ first metacarpal bone, $2 \mathrm{nd} \mathrm{MC}=$ second metacarpal bone

agreement, $0.41-0.6$ moderate agreement, $0.61-0.8$ substantial agreement and $0.81-1$ almost perfect agreement. According to Rosner [23], the quality of inter-rater reliability by means of ICC was classified as following: $>0.75$ excellent, $0.4-0.75$ fair to good, and $<0.4$ poor.

Gender differences of the anatomical characteristics of the $\mathrm{CMC}$ joint were assessed using an independent $t$ test. A $p$ value of $<0.05$ was considered statistically significant. For all analyses, a statistical software (SPSS for Windows, release 17.0; SPSS, Chicago, IL, USA) was used.

\section{Results}

We scanned $44 \%(15 / 34)$ dominant thumbs and $56 \%$ $(19 / 34)$ non-dominant thumbs. In $44 \%$ of individuals $(15 / 34)$ the thumb is particularly used in labor activities, such as typing $(29 \% ; 10 / 34)$, physiotherapy $(9 \% ; 3 / 34)$,

Table 1 Qualitative characteristics of the carpometacarpal (CMC) ligament complex of the thumb in asymptomatic volunteers

\begin{tabular}{|c|c|c|c|c|c|}
\hline & Ligament & Visibility & Low SI & Increased SI & Striated SI \\
\hline \multirow[t]{4}{*}{ Reader 1} & AOL/beak & $97(33 / 34)$ & $36(12 / 33)$ & $27(9 / 33)$ & $36(12 / 33)$ \\
\hline & POL & $100(34 / 34)$ & $6(2 / 34)$ & $65(22 / 34)$ & $29(10 / 34)$ \\
\hline & IML & $100(34 / 34)$ & $3(1 / 34)$ & $6(2 / 34)$ & $91(31 / 34)$ \\
\hline & DRL & $97(33 / 34)$ & $33(11 / 33)$ & $58(19 / 33)$ & $9(3 / 33)$ \\
\hline \multirow[t]{4}{*}{ Reader 2} & AOL/beak & $97(33 / 34)$ & $42(14 / 33)$ & $27(9 / 33)$ & $30(10 / 33)$ \\
\hline & POL & $100(34 / 34)$ & $12(4 / 34)$ & $74(25 / 34)$ & $15(5 / 34)$ \\
\hline & IML & $100(34 / 34)$ & $12(4 / 34)$ & $12(4 / 34)$ & $76(26 / 34)$ \\
\hline & DRL & $97(33 / 34)$ & $18(6 / 33)$ & $64(21 / 33)$ & $18(6 / 33)$ \\
\hline
\end{tabular}

Data are percentage with absolute numbers in parentheses, calculated from the number of visible ligaments

AOL/beak anterior oblique ligament, $P O L$ posterior oblique ligament, $I M L$ intermetacarpal ligament, $D R L$ dorsoradial ligament

SI Signal intensity in intermediate-weighted fat-saturated images, low as the normal low signal of ligaments and tendons, increased compared to the normal low signal of ligaments and tendons, striated with lamellar low and increased SI 


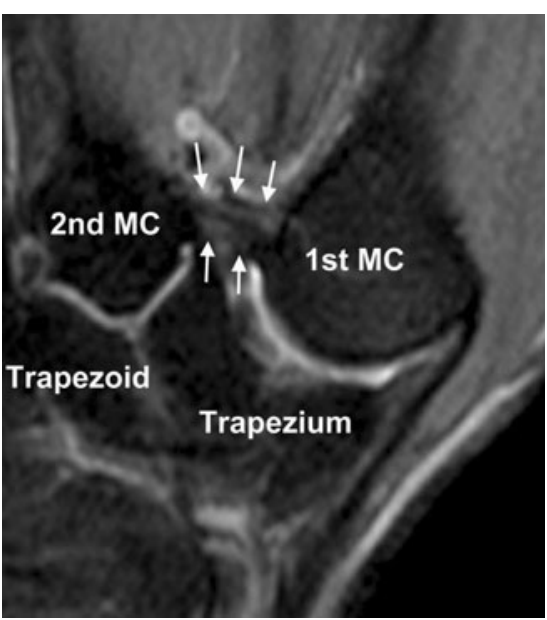

Fig. 5 Coronal intermediate-weighted fat-saturated image $(2,039 / 25 \mathrm{~ms})$ shows the striated appearance of the IML (arrows) of a 26-year-old healthy female volunteer. 1 st $\mathrm{MC}=$ first metacarpal bone, 2 nd $\mathrm{MC}=$ second metacarpal bone, $\mathrm{IML}=$ intermetacarpal ligament

and handcraft $(6 \% ; 2 / 34)$. In $56 \%$ of individuals (19/34), the thumb is not exposed to specific labor activities.

\section{Qualitative analysis}

A summary of the qualitative results of both readers is shown in Table 1.

The ligaments of the $\mathrm{CMC}$ joint were visible in all volunteers for the POL and IML and in all but one for the AOL and DRL with a perfect $k$ value of 1 . On intermediate-weighted FS images the POL and DRL were commonly of increased SI. The IML had a striated appearance (Fig. 5). The AOL showed a variable SI distribution. The inter-observer agreement $(K)$ of the SI ranged from 0.3 to 0.5 .

The chondral surface of the CMC joint showed in $26 \% / 35 \%$ (reader $1 /$ reader $2 ; 9 / 34$ reader $1 ; 12 / 34$ reader 2 ) an inhomogeneous SI in intermediate-weighted FS images and in $15 \% / 29 \%(5 / 34$ reader $1 ; 10 / 34$ reader 2$)$ chondral defects were present ( $\mathrm{k}$ 0.4, Fig. 6).
A minor amount of joint fluid was observed in most cases ( $88 \%, 30 / 34$ for both readers; $\kappa$ 1; Fig. 7), a joint distension due to a substantial effusion was never seen in our study group. A minority of four volunteers $(12 \%$ in both readers, $k 1)$ showed no joint fluid.

\section{Quantitative analysis}

The thickness of the ligaments is presented in Table 2. The AOL was significantly thicker in men $(1.7 \mathrm{~mm} \pm 0.6 / 1.7 \mathrm{~mm}$ \pm 0.5 , reader $1 /$ reader 2$)$ than in women $(1.2 \mathrm{~mm} \pm$ $0.6 / 1.4 \mathrm{~mm} \pm 0.6 ; p=0.02)$ for reader $1(p=0.08$ reader 2$)$, while the IML was not substantially $(p=0.07$ in both readers) thicker in men $(3.2 \mathrm{~mm} \pm 1.1 / 3.3 \mathrm{~mm} \pm 0.7)$ than in women $(2.6 \mathrm{~mm} \pm 0.9 / 2.8 \mathrm{~mm} \pm 0.9)$. The POL $(p=0.8 / 0.3$ reader $1 /$ reader 2$)$ and DRL $(p=0.2 / 0.4)$ showed no significant gender differences in both readers. The overall ICC ranged from $0.2-0.8$, while correlation for the AOL was good with an ICC of 0.8 .

A mean dorsal subluxation of $1.8 \mathrm{~mm} \pm 1.0 / 2.0 \mathrm{~mm} \pm$ 1.0 and radial subluxation of $2.8 \mathrm{~mm} \pm 1.5 / 3.4 \mathrm{~mm} \pm 1.6$ of the metacarpal base was found (Fig. 4). Radial subluxation was significantly larger in men $(3.4 \mathrm{~mm} \pm$ $1.5 / 3.7 \mathrm{~mm} \pm 1.6)$ than in women $(2.2 \mathrm{~mm} \pm 1.3 / 3.1 \mathrm{~mm}$ $\pm 1.5 ; p=0.02 / 0.2$ ) for reader 1 (Table 3 ). No subluxation in palmar or ulnar direction was seen. The ICC ranged from $0.5-0.8$ with a good correlation for assessment of the radial subluxation $(0.8)$.

\section{Discussion}

In our study, the ligaments of the $\mathrm{CMC}$ joint (AOL/beak, POL, IML, and DRL) were of variable SI ranging from low to increased SI. Even a striated appearance was observed, contrary to the low SI of the CMC ligaments reported in previous studies $[3,5]$. In our study, the low SI was only evident in a minority of volunteers, with the least number of low SI cases for the POL and IML. In addition, the POL
Fig. 6 a Coronal $(2,039 / 25)$ and b sagittal $(2,000 / 24 \mathrm{~ms})$ intermediate-weighted fat-saturated MR images show loss of cartilage (in between the arrows) of the first metacarpal base and the trapezium in a 36-year-old asymptomatic volunteer. 1 st $\mathrm{MC}=$ first metacarpal bone
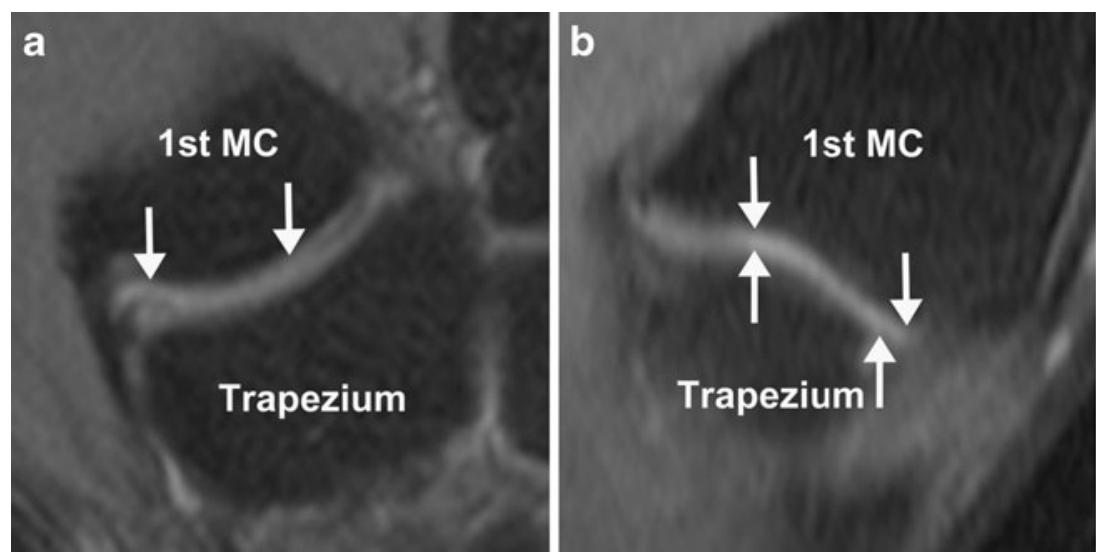


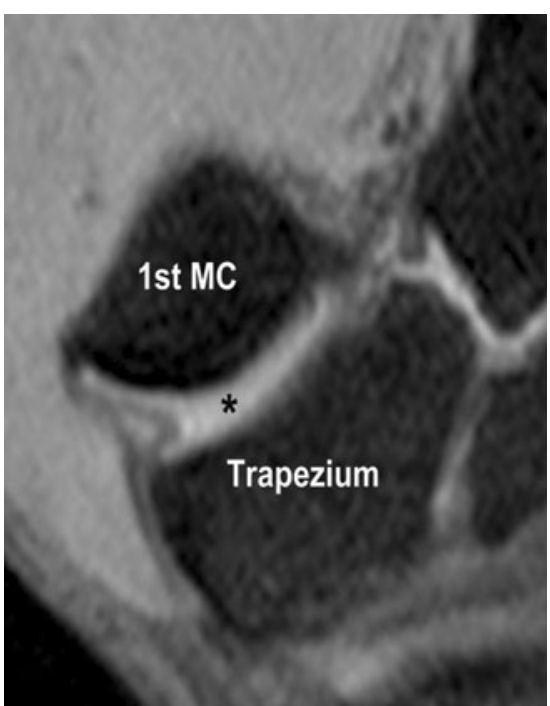

Fig. 7 Coronal intermediate-weighted fat-saturated MR image $(2,039 / 25$ $\mathrm{ms}$ ) shows minor joint fluid (asterisk) of the CMC joint of the thumb in a 33 -year-old asymptomatic volunteer. 1 st $\mathrm{MC}=$ first metacarpal bone

showed an increased SI (65-74 \%) and the IML a striated appearance (76-91\%) in the majority of individuals.

In previous imaging studies with MR and ultrasound [5, 17], the deep and superficial layers of the AOL could not be differentiated due to the small dimension and the close relationship without interposing tissue. A recently published study [3] distinguished these two bundles using MR arthrography. In our study, in which a dedicated extremity scanner was used without administration of intraarticular contrast, we found a striated $\mathrm{AOL}$ in about $1 / 3$ of the cases, which might be due to the layered architecture of this ligament (Fig. 8). The variance of ligament SI of the AOL and POL in our study compared to the reported low SI of ligaments might be related to the magic angle effect, as the echo times of the coronal and sagittal intermediated-balanced FS sequences were of 25 and $24 \mathrm{~ms}$, respectively. Because of the horizontal orientation of the IML, the mostly striated appearance does not assume to be related to the magic angle effect, and instead might be due to the ligament morphology $[30,31]$.
In MR imaging of the thumb, we were consistently able to outline four ligaments - the AOL/beak on the volar side, the POL and DRL on the dorsal side, and the IML at the ulnar aspect of the joint. Just in one female volunteer we could not visualize the AOL, which might be due to its intracapsular location or an old rupture, even though the volunteers negated a prior trauma to the thumb. The DRL was well delineated, which might be due to the superficial location adjacent to the insertion of the abductor pollicis longus tendon (Fig. 3). However, in one case the DRL was not present. The IML was well visualized in all individuals due to the extracapsular location. In anatomic dissections several, ligaments of the CMC joint were described [1, 10-18]. The capsular AOL and POL and the extracapsular IML have been described as three major components $[4,7$, $24,25]$. There is still controversy about which ligament is the key stabilizer of the CMC joint. Some believe that the AOL is the most important stabilizer [10-12, 26, 27] while others believe that the dorsal ligamentous structures are more important [4, 7, 13-15]. Xu et al. [28] found an equal stiffness for the AOL, POL, and DRL in a computer model study.

The extracapsular UCL is a thin, narrow structure overlapping the AOL and cannot be distinguished from the AOL in ultrasound or MR imaging [17], this is in accordance to our experience. Therefore, the UCL was not analyzed in this study.

The thickness differed in all four ligaments, with the IML being the thickest of all with $2.9 \mathrm{~mm}$ to $3.1 \mathrm{~mm}$ and the DRL being the thinnest with $1.2 \mathrm{~mm}$ to $1.4 \mathrm{~mm}$. The thickness of the AOL with $1.4 \mathrm{~mm}$ to $1.6 \mathrm{~mm}$ corresponded very well to the mean thickness of $1.28 \mathrm{~mm}$ in an ultrasound study with ten cadavers and ten volunteers [17]. Our measurement of the DRL thickness $(1.2 \mathrm{~mm} \pm 0.5 / 1.4 \mathrm{~mm} \pm 0.5)$ and the AOL thickness $(1.4 \mathrm{~mm} \pm 0.6 / 1.6 \mathrm{~mm} \pm 0.6)$ is very similar compared to an anatomy dissection of fresh-frozen cadavers (0.9 $\mathrm{mm} \pm 0.2$ and $2.0 \mathrm{~mm} \pm 0.1$, respectively) [16]. The significant gender difference in thickness of the AOL in our study might be due to general tenderness in anatomic structures in women. The thinner AOL in women might be associated with a higher prevalence of thumb CMC

Table 2 Quantitative analysis of the ligament thickness of the CMC joint of the thumb in asymptomatic volunteers

\begin{tabular}{lllllllll}
\hline Parameter & Reader 1 & Women & Men & $p$ value & Reader 2 & Women & Men & $p$ value \\
\hline AOL/beak & $1.4 \pm 0.6$ & $1.2 \pm 0.6$ & $1.7 \pm 0.6$ & $0.02 *$ & $1.6 \pm 0.6$ & $1.4 \pm 0.6$ & $1.7 \pm 0.5$ \\
POL & $2.3 \pm 0.6$ & $2.3 \pm 0.6$ & $2.3 \pm 0.5$ & 0.8 & $1.9 \pm 0.5$ & $2.0 \pm 0.5$ & $1.8 \pm 0.5$ \\
IML & $2.9 \pm 1.0$ & $2.6 \pm 0.9$ & $3.2 \pm 1.1$ & 0.07 & $3.1 \pm 0.8$ & $2.8 \pm 0.9$ & $3.3 \pm 0.7$ & 0.3 \\
DRL & $1.2 \pm 0.5$ & $1.3 \pm 0.5$ & $1.1 \pm 0.4$ & 0.2 & $1.4 \pm 0.5$ & $1.3 \pm 0.4$ & $1.5 \pm 0.6$ & 0.4 \\
\hline
\end{tabular}

Data are mean thickness and standard deviation in millimeter

* $p$ value $<0.05$ was considered statistically significant

$A O L /$ beak anterior oblique ligament, $P O L$ posterior oblique ligament, $I M L$ intermetacarpal ligament, $D R L$ dorsoradial ligament 
Table 3 Quantitative analysis of the alignment of the CMC joint of the thumb in asymptomatic volunteers

\begin{tabular}{lllllllll}
\hline Parameter & Reader 1 & Women & Men & $p$ value & Reader 2 & Women & Men & $p$ value \\
\hline Dorsal subluxation & $1.8 \pm 1.0$ & $2.0 \pm 0.7$ & $1.7 \pm 1.3$ & 0.4 & $2.0 \pm 1.0$ & $2.1 \pm 0.9$ & $1.9 \pm 1.1$ & 0.6 \\
Radial subluxation & $2.8 \pm 1.5$ & $2.2 \pm 1.3$ & $3.4 \pm 1.5$ & $0.02 *$ & $3.4 \pm 1.6$ & $3.1 \pm 1.5$ & $3.7 \pm 1.6$ & 0.2 \\
\hline
\end{tabular}

Data are mean distance and standard deviation in millimeters

* $p$ value $<0.05$ was considered statistically significant.

Alignment of the $\mathrm{CMC}$ joint was quantified by measuring the distance between the cartilage-covered portion parallel to the joint surface of the base of the first metacarpal bone and the trapezium in coronal and sagittal planes

osteoarthritis in females compared to males [32], although Imaeda [1] described a thickened AOL of severely osteoarthritic patients in a cadaver study.

The thickness of all CMC joint ligaments varied considerably in our study. Therefore, the thickness of these ligaments is not necessarily a sign of ligament injury or early osteoarthritis. Scarring of ligaments or a recent injury may lead to increased SI and thickening of the CMC ligaments. However, we also found increased SI and ligament thickening in healthy volunteers, so these signs should not be misinterpreted as a clear sign of abnormality when analyzing MR images.

In our study, inhomogeneous SI and irregularities of the cartilage of the $\mathrm{CMC}$ joint were detected in one-third of the individuals. These findings occur in asymptomatic volunteers.

In MR imaging obtained in the resting position of the thumb, the base of the first metacarpal bone showed a subluxation in the dorsoradial direction in relation to the trapezium in all volunteers in our study (Fig. 4).

According to the literature, recognizing the dorsoradial joint incongruity is crucial in observing posttraumatic changes, and also severe osteoarthritis is associated with joint subluxation [1]. We found a mean radial subluxation of 2.8$3.4 \mathrm{~mm}$ and a dorsal subluxation of $1.8-2.0 \mathrm{~mm}$. This is in accordance to an X-ray study of 69 healthy volunteers with a range of radial subluxation of $1.72-8.69 \mathrm{~mm}$ [29]. This amount of subluxation should not be misinterpreted as a possible sign of prior trauma or osteoarthritis in a resting thumb position. The CMC joint is only congruent in thumb opposition $[4,7]$. MR imaging of the CMC joint with the thumb in opposition is not comfortable. However, this position might help to differentiate a position-related subluxation from an underlying posttraumatic or disease-related subluxation. A statistically significant larger radial subluxation was observed in men compared to women in our study. We found a minor amount of joint fluid in most healthy volunteers (Fig. 7), so this should not be misinterpreted as an early sign of osteoarthritis or synovitis.

The following limitations of our study have to be acknowledged. A rather small number of 34 volunteers were evaluated in this study. We scanned the thumb in a resting position and characterized the CMC ligaments in routine thumb imaging without special angulated planes to this joint. Due to the wrapping configuration of the ligaments, the detection might be more difficult in non joint-orthogonal planes. Due to the small dimensions of the analyzed ligaments, the quantitative measurements might be prone to higher variability, although interobserver reliability was on average acceptable. As this is an anatomical study of healthy volunteers, we did not correlate our findings with surgical findings or cadaveric dissections.
Fig. 8 a Sagittal intermediateweighted fat-saturated (2,000/24 $\mathrm{ms})$ and $\mathbf{b}$ T1-weighted (598/14 ms) MR images show a striated appearance of the anterior oblique ligament/beak ligament of the CMC joint of the thumb, deep (short arrows) and superficial (long arrows) bundles in a 32-year-old asymptomatic volunteer. $*=\mathrm{POL}$ (posterior oblique ligament), $1 \mathrm{st} \mathrm{MC}=$ first metacarpal bone
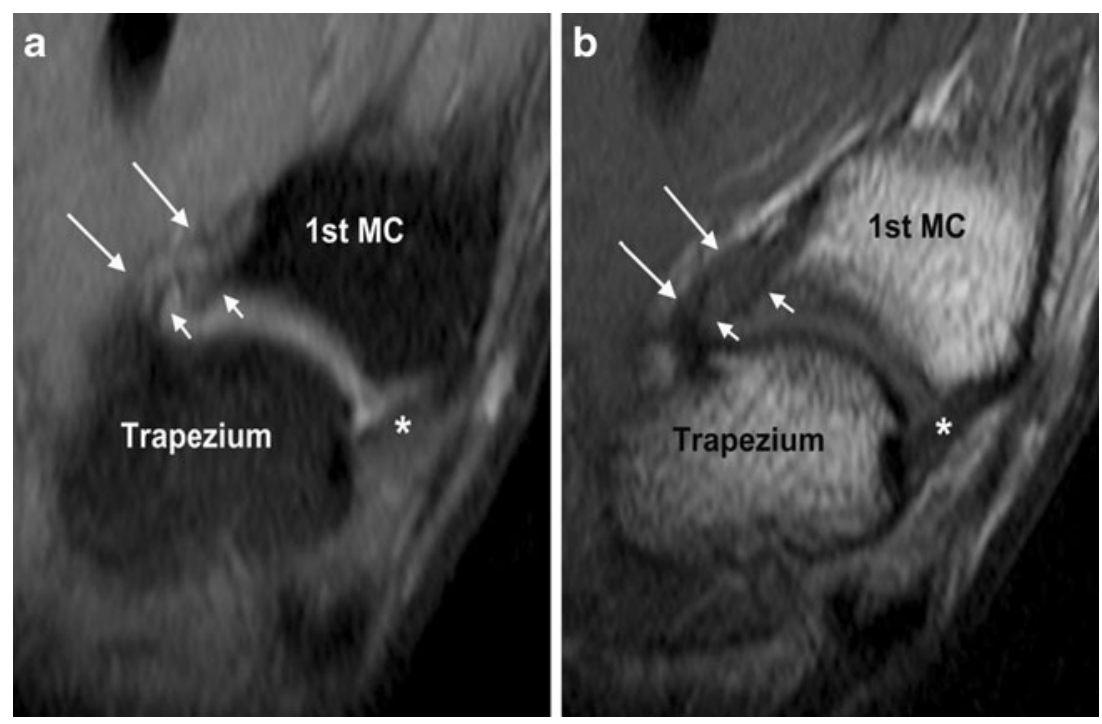
Detecting and characterizing the morphology of the CMC ligament complex of the thumb in MR images may be helpful for hand surgeons when planning surgery in patients with CMC joint instability. Detailed information about the ligament morphology with variable signal intensities and radiodorsal subluxation of the joint in healthy volunteers is presented, which may be useful for MR evaluation of carpometacarpal diseases of the thumb.

In summary, radial and dorsal subluxation of the CMC joint can be a normal finding in a resting position at MR imaging. The CMC ligaments of the thumb showed a considerable variability in MR imaging in healthy volunteers; the IML is typically striated. Thickness of the AOL is typically less than $2.2 \mathrm{~mm}$, and of the POL typically less than $2.9 \mathrm{~mm}$. Knowledge of the ligament integrity and joint alignment is helpful in analyzing MR images of the CMC joint in thumb injuries and osteoarthritis.

Conflict of interest The authors declare that they have no conflicts of interest.

\section{References}

1. Imaeda T, An KN, Cooney WP, Linscheid R. Anatomy of trapeziometacarpal ligaments. J Hand Surg. 1993;18A:226-31.

2. Lane LB, Henley DH. Ligament reconstruction of the painful, unstable, nonarthritic thumb carpometacarpal joint. J Hand Surg. 2001;26 A:686-91

3. Cardoso FN, Kim HJ, Albertotti F, Botte MJ, Resnick D, Chung CB. Imaging the ligaments of the trapeziometacarpal joint: MRI compared with MR arthrography in cadaveric specimens. AJR. 2009;192:W13-9.

4. Edmunds JO. Current concepts of the anatomy of the thumb trapeziometacarpal joint. J Hand Surg. 2011;36A:170-82.

5. Connell DA, Pike J, Koulouris G, van Wettering N, Hoy G. MR imaging of thumb carpometacarpal joint ligament injuries. J Hand Surg Br. 2004;29B(1):46-54.

6. Bosmans B, Verhofstad MHJ, Gosens T. Traumatic thumb carpometacarpal joint dislocation. J Hand Surg. 2008;33A:43841.

7. Edmunds JO. Traumatic dislocations and instability of the trapeziometacarpal joint of the thumb. Hand Clin. 2006;22:36592.

8. Fotiadis E, Svarnas T, Lyrtzis C, Papadopoulos A, Akritopoulos P, Chalidis B. Isolated thumb carpometacarpal joint dislocation: a case report and review of the literature. J Orthop Surg Res. 2010;5:16

9. Brownlie C, Anderson D. Bennett fracture dislocation - review and management. Aus Fam Physician. 2011;40(6):394-6.

10. Pellegrini Jr VD, Olcott CW, Hollenberg G. Contact patterns in the trapeziometacarpal joint: the role of the palmar beak ligament. J Hand Surg. 1993;18a:238-44.
11. Napier JR. The form and function of the carpometacarpal (CMC) joint. J Anat. 1955;89:362-9.

12. Bettinger PC, Linscheid RL, Berger RA, Cooney WP, Kai-Nan A. An anatomic study of the stabilizing ligaments of the trapezium and trapeziometacarpal joint. J Hand Surg. 1999;24A:786-98.

13. Strauch RJ, Behrman MJ, Rosenwasser MP. Acute dislocation of the carpometacarpal joint of the thumb: an anatomic and cadaver study. J Hand Surg Am. 1994;19:93-8.

14. Van Brenk B, Richards RR, Mackay MB, Boynton EL. A biomechanical assessment of ligaments preventing dorsoradial subluxation of the trapeziometacarpal joint. J Hand Surg. 1998;23A:60711.

15. Cooney W, Chao E. Biomechanical analysis of static forces in the thumb during hand function. J Bone Joint Surg. 1977;59a:27-36.

16. Nanno M, Burford WL, Patterson RM, Andersen CR, Viegas SF. Three-dimensional analysis of the ligamentous attachments of the first carpometacarpal joint. J Hand Surg. 2006;31 A:1160-70.

17. Gondim Teixeira PA, Omoumi P, Trudell DJ, Ward SR, Blum A, Resnick DL. High-resolution ultrasound evaluation of the trapeziometacarpal joint with emphasis on the anterior oblique ligament (beak ligament). Skeletal Radiol. 2011;40:897-904.

18. Doerschuk SH, Hicks DG, Chinchilli VM, Pellegrini VD. Histopathology of the palmar beak ligament in trapeziometacarpal osteoarthritis. J Hand Surg. 1999;24a:496-504.

19. Husarik DB, Saupe N, Pfirmann CWA, Jost B, Hodler J, Zanetti M. Ligaments and plicae of the elbow: normal MR imaging variability in 60 asymptomatic subjects. Radiology. 2010;257:185-94.

20. Mengiardi B, Pfirrmann CWA, Vienne P, Hodler J, Zanetti M. Medial collateral ligament complex of the ankle: MR appearance in asymptomatic subjects. Radiology. 2007;242:817-24.

21. Pellegrini Jr VD. Pathomechanics of the thumb trapeziometacarpal joint. Hand Clin. 2001;17:175-84.

22. Landis JR, Koch GG. The measurement of observer agreement for categorical data. Biometrics. 1977;33(1):159-74.

23. Rosner BA. Fundamentals of Biostatistics 5th Edition. Duxbury 2000

24. Bojsen-Moller F. Osteoligamentous guidance of the movements of the human thumb. Am J Anat. 1976;147:71-80.

25. Pagalidis T, Kuczynski K, Lamb DW. Ligamentous stability of the base of the thumb. Hand. 1981;13:29-35.

26. Eaton RG, Littler JW. A study of the basal joint of the thumb, treatment of its disabilities by fusion. J Bone Joint Surg. 1969;51a:661-8.

27. Eaton RG, Littler JW. Ligament reconstruction of the painful thumb carpometacarpal joint. J Bone Joint Surg. 1973;55a(8):802-6.

28. $\mathrm{Xu} \mathrm{L,} \mathrm{Strauch} \mathrm{RJ,} \mathrm{Ateshian} \mathrm{GA,} \mathrm{Pawluk} \mathrm{RJ,} \mathrm{Mow} \mathrm{VC,}$ Rosenwasser MP. Topography of the osteoarthritic thumb carpometacarpal joint and its variation with regard to gender, age, site and osteoarthritic stage. J Hand Surg. 1998;23a:454-64.

29. Wolf JM, Oren TW, Ferguson B, Williams A, Peterson B. The carpometacarpal stress view radiograph in the evaluation of trapeziometacarpal joint laxity. J Hand Surg Am. 2009;34(8):14026.

30. Hayes CW, Paralleda JA. The magic angle effect in musculoskeletal MR imaging. Top Magn Reson Imaging. 1996;8(1):51-6.

31. Bydder M, Rahal A, Fullerton GD, Bydder GM. The magic angle effect: a source of artifact, determinant of image contrast, and technique for imaging. J Magn Reson Imaging. 2007;25(2):290300 .

32. Badia A. Arthroscopy of the trapeziometacarpal and metacarpophalangeal joints. J Hand Surg Am. 2007;32(5):707-24. 